increase noticeable between ten and fifteen days after the passage of large spots through the central meridian of the sun is still uncertain and may be purely incidental.

Another point of interest was the investigation of magnetic storms. Some time ago A. Corlin ${ }^{3}$ found, that in Abisko (northern Sweden) the cosmic ray intensity increases after the beginning of a magnetic storm, especially if this is accompanied by a so-called magnetic 'impetus'.

From September 1931 to March 1933 twenty-four magnetic storms were observed; the magnetic data were taken from the records of the Geomagnetic Station at Vienna (Auhof). With the apparatus screened with $10 \mathrm{~cm}$. lead on all sides, we obtained an average cosmic ray ionisation of $2.751 \mathrm{~J}$ in the ten hours preceding the storm and $2 \cdot 743 \mathrm{~J}$ in the ten hours after the beginning of each magnetic storm. With no lead screen on top of the apparatus the ten-hour means were $4.547 J$ before and $4.536 J$ after the beginning of the magnetic storm.

Thus our observations indicate that a slight decrease of the cosmic ray ionisation (by $0.008 \mathrm{~J}$ with lead screen all around and $0 \cdot 011 J$ with no lead on top of the apparatus) occurs after the beginning of the magnetic disturbance. This is in apparent contradiction to Dr. Corlin's results in northern Sweden. On the other hand, the effect in our latitudes is, of course, by no means comparable with that in lat. $68^{\circ} \mathrm{N}$. It seems quite possible that an increase of the cosmic ray intensity in the far north is accompanied by a decrease in Central Europe. Dr. Corlin agrees with us on this point. It can be expected that the simultaneous observations carried out by Dr. Corlin in Abisko and by us on the Hafelekar will clear up this point.

It can be said that our results are an additional argument for the corpuscular theory of the cosmic radiation, or at least of a part of it. The strongest proof so far is, of course, the so-called latitude effect, discovered by $J$. Clay on his journeys from Holland to Java and back (1927-1929). Lemaître and Vallarta ${ }^{4}$ erroneously ascribed the discovery of the latitude effect to A. H. Compton. The great merit of Compton's world-wide survey of the intensity distribution of cosmic radiation is by no means lessened by stating that not he, but Clay, found first that the intensity of cosmic rays decreases towards the equator.

VICTOR F. Hess.

R. Steinmaurer.

Institut für Strahlenforschung, Universität, Innsbruck. Sept. 18.

1 V. F. Hess, Terr. $M a g ., 137,399 ; 1932$. V. F. Hess and R. Steinmaurer, Sitz. Berichte Berlin, Akad. d. Wiss., 15, 521; 1933.

2 O. Freytag, Gerlands Beitr. z. Geophys., 37, Nr. 1; 1933.

3 A. Corlin, Lund Observatory Bulletin, Nr. 1; 1931.

- Phys. Rev., 43, 87 ; 1933.

\section{Production of High Magnetic Fields at Low Temperatures}

THE use of a supra-conductor (therefore completely free from Joule heating) has been more than once suggested for the production of magnetic fields at low temperatures. The magnetic field obtainable by this means is limited by the magnetic threshold value at which supra-conductivity ceases. Still, considerable fields can be obtained by the use of alloys (investigated in Leyden ${ }^{1}$ ) the threshold value of which is
22,000 gauss at $2^{\circ} \mathrm{K}$., a strength which is sufficient for many experiments.

The chief remaining difficulty lies in the heat conductivity of the leads to the supra-conducting coil. This problem of heat conduction through the leads can be eliminated by transferring the necessary energy for the magnetic field by induction. The suggested arrangement is similar in principle to a transformer, the primary circuit of which is normally conducting and the secondary circuit of which is supra-conducting. The primary circuit eonsists of a D.C. source and the primary of the transformer; the supra-conducting secondary circuit consists of a secondary with a few turns of large radius and a solenoid with many narrow turns for producing the high field. On closing the primary circuit the magnetic energy transferred to the secondary is shared with the solenoid. With this arrangement, one produces, to some extent, condensation of the lines of force. Calculation shows that for given geometrical dimensions there is an optimum ratio for the number of turns in the secondary coil to that in the solenoid. In this way, within the limits of the usual dimensions of an apparatus, it is easily possible with a primary field of about I,000 gauss to obtain a field of 22,000 gauss in the space of a few cubic centimetres.

The method should be specially suitable whenever it is desired to produce fairly high magnetic fields at low temperatures in not too large a volume, as, for example, in the production of extremely low tem. peratures by the adiabatic demagnetisation of paramagnetic substances ${ }^{2}$. According to the experiments of Kürti and Simon ${ }^{3}$, Giauque and MacDougall ${ }^{4}$ and de Haas and his co-workers ${ }^{5}$, it should be possible, with the above arrangement, to obtain temperatures below $0 \cdot 1^{\circ} \mathrm{K}$. from a starting point of $1^{\circ} \mathrm{K}$. Since heat conductivity along the current leads is eliminated, and since heat capacities at helium temperatures are so minute, a few cubic centimetres of liquid helium should suffice to cool the whole arrangement.

Experiments with an apparatus embodying the above methods are being made in this laboratory.

Clarendon Laboratory, K. Meindelssohn.

Oxford.

Sept. 28.

${ }^{1}$ W. J. de Haas and J. Voogd, Comm. Leiden, No. 214b ; 1931.

${ }^{2}$ P. Debye, Ann. Phys. (4) 81,$1154 ; 1926$. W. F. Giauque, J. Amer. Chem. Soc., 49, $1864 ; 1927$.

${ }^{3}$ N. Kürti and F. Simon, Naturwiss., 21, 178 ; 1933.

4 W. F. Giauque, D. P. MacDougall, Phys. Rev., 44, 235 ; 1933.

${ }^{5}$ W. J. de Haas, NATURe, 132, 372, Sept. 9, 1933.

\section{Structure of Emulsoid Sol Particles and their Hydration Film}

Tere powder-photographs of cellulose, starch and similar substances have been interpreted as originating from the crystal structure of those substances. In the course of an investigation with $\mathrm{X}$-rays into the structure of the hydration film of emulsoid sol particles, we arrived at provisional results which indicate that this interpretation is liable to be erroneous.

It seems to us at present highly probable that at least the greater part-perhaps almost all-of the powder lines of some of the said substances is due to crystal structure, not of the substance itself, but of the hydration film surrounding its particles (that film has been called 'concrete' before).

A kind of regularity in the marshalling of the 